\title{
EULER FLOW SOLUTIONS FOR TRANSONIC SHOCK WAVE-BOUNDARY LAYER INTERACTION
}

\author{
BARRY KOREN \\ Centre for Mathematics and Computer Science, P.O. Box 4079, 1009 AB Amsterdam, The Netherlands
}

\begin{abstract}
SUMMARY
Steady 2D Euler flow computations have been performed for a wind tunnel section, designed for research on transonic shock wave-boundary layer interaction. For the discretization of the steady Euler equations, an upwind finite volume technique has been applied. The solution method used is collective, symmetric point Gauss-Seidel relaxation, accelerated by non-linear multigrid. Initial finest grid solutions have been obtained by nested iteration. Automatic grid adaptation has been applied for obtaining sharp shocks. An indication is given of the mathematical quality of four different boundary conditions for the outlet flow. Two transonic flow solutions with shock are presented: a choked and a non-choked flow. Both flow solutions show good shock capturing. A comparison is made with experimental results.
\end{abstract}

KEY WORDS Steady Euler equations Transonic flows Multigrid methods Boundary conditions

\section{INTRODUCTION}

An important physical feature for the design of transonic aerofoils is the interaction between the possible shock wave(s) at the aerofoil and the boundary layers along the aerofoil. In transonic aerodynamics a lot of work, both experimental and theoretical, is devoted to this so-called transonic shock wave-boundary layer interaction. At the Delft University of Technology, Faculty of Aerospace Engineering, a transonic wind tunnel section has been designed and constructed ${ }^{1}$ for performing measurements on this phenomenon. ${ }^{2}$ Limited accessibility to the flow in the wind tunnel section inhibits measurements throughout the entire flow field. However, knowledge of the entire flow field is important for redesign purposes. This situation motivated a computation of the entire flow field.

As a suitable flow model, the steady 2D Euler equations have been chosen. The Euler equations have been chosen because (in the first instance) only inviscid flow solutions with (possibly occurring) rotation are of interest. The use of a steady flow model is motivated by the fact that the main flow in the wind tunnel section is steady. Further, the use of a 2D flow model is motivated by the fact that the wind tunnel section has a curved lower and upper wall, and flat parallel side walls.

\section{COMPUTATIONAL METHOD}

\subsection{Discretization method}

The steady $2 \mathrm{D}$ Euler equations can be written on the domain $\Omega \subset \mathbb{R}^{2}$ as

$$
\frac{\partial \mathbf{f}(\mathbf{q})}{\partial x}+\frac{\partial \mathbf{g}(\mathbf{q})}{\partial y}=\mathbf{0}
$$


with

$$
\mathbf{q}=\left[\begin{array}{l}
\rho \\
\rho u \\
\rho v \\
\rho e
\end{array}\right], \quad \mathbf{f}(\mathbf{q})=\left[\begin{array}{l}
\rho u \\
\rho u^{2}+p \\
\rho u v \\
\rho u(e+p / \rho)
\end{array}\right], \quad \mathbf{g}(\mathbf{q})=\left[\begin{array}{l}
\rho v \\
\rho u v \\
\rho v^{2}+p \\
\rho v(e+p / \rho)
\end{array}\right]
$$

Here $\mathbf{q}$ is the state vector of conservative quantities, and $\mathbf{f}$ and $\mathbf{g}$ are the so-called flux vectors. The primitive quantities used here are the density $\rho$, the velocity components $u$ and $v$ and the pressure $p$. For a perfect gas the total energy $e$ is related to the primitive quantities as

$$
e=\frac{1}{\gamma-1} \frac{p}{\rho}+\frac{1}{2}\left(u^{2}+v^{2}\right)
$$

where $\gamma$ is the ratio of specific heats.

To allow solutions with discontinuities, the Euler equations are discretized in the integral form

$$
\int_{a \Omega^{*}}(\mathbf{f}(\mathbf{q}) \cos \phi+\mathbf{g}(\mathbf{q}) \sin \phi) \mathrm{d} s=\mathbf{0},
$$

where $\Omega^{*}$ is an arbitrary subregion of $\Omega, \partial \Omega^{*}$ is the boundary of $\Omega^{*}$, and $\cos \phi$ and $\sin \phi$ are the $x$ and $y$-component respectively of the outward unit normal on $\partial \Omega^{*}$. A straightforward and simple discretization of (4) is obtained by subdividing $\Omega$ into disjunct subregions $\Omega_{i, j}$ (the finite volumes) and requiring that

$$
\int_{\partial \Omega_{i, j}}(\mathbf{f}(\mathbf{q}) \cos \phi+\mathbf{g}(\mathbf{q}) \sin \phi) \mathrm{d} s=\mathbf{0}
$$

for each finite volume separately.

Using the rotational invariance of the Euler equations,

$$
\mathbf{f}(\mathbf{q}) \cos \phi+\mathbf{g}(\mathbf{q}) \sin \phi=\mathbf{T}^{-1}(\phi) \mathbf{f}(\mathbf{T}(\phi) \mathbf{q}),
$$

where $\mathbf{T}(\phi)$ is the rotation matrix

$$
\mathbf{T}(\phi)=\left[\begin{array}{ccll}
1 & 0 & 0 & 0 \\
0 & \cos \phi & \sin \phi & 0 \\
0 & -\sin \phi & \cos \phi & 0 \\
0 & 0 & 0 & 1
\end{array}\right]
$$

(5) can be rewritten as

$$
\int_{c \Omega_{i, j}} \mathbf{T}^{-1}(\phi) \mathbf{f}(\mathbf{T}(\phi) \mathbf{q}) \mathrm{d} s=\mathbf{0}
$$

As finite volumes we consider (arbitrarily shaped) quadrilaterals. The subdivision into quadrilaterals is such that $\Omega_{i \pm 1, j}$ and $\Omega_{i, j \pm 1}$ are the neighbouring volumes of $\Omega_{i, j}$ (Figure 1(a)).

Crucial in the discretization is the evaluation of the flux vector along $\partial \Omega_{i, j}$. Along each finite volume wall separately we assume the flux vector to be constant, and we assume it to be determined by a uniformly constant left and right state only. Hence (8) becomes

$$
\mathbf{F}_{i+1 / 2, j}-\mathbf{F}_{i-1 / 2, j}+\mathbf{F}_{i, j+1 / 2}-\mathbf{F}_{i, j-1 / 2}=\mathbf{0},
$$

with

$$
\mathbf{F}_{i+1 / 2, j}=\mathbf{T}^{-1}\left(\phi_{i+1 / 2, j}\right) \mathbf{f}\left(\mathbf{T}\left(\phi_{i+1 / 2, j}\right) \mathbf{q}_{i+1 / 2, j}^{l}, \mathbf{T}\left(\phi_{i+1 / 2, j}\right) \mathbf{q}_{i+1 / 2, j}^{r}\right) l_{i+1 / 2, j}
$$




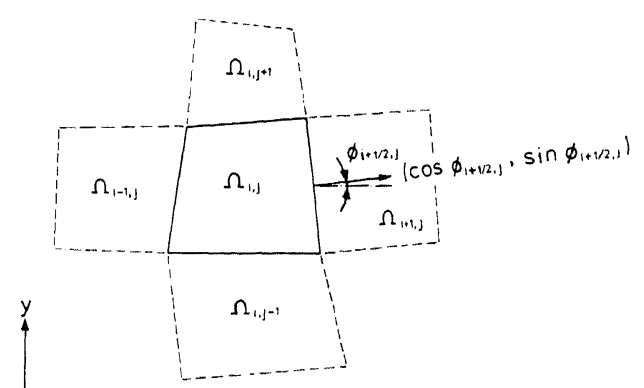

(a)

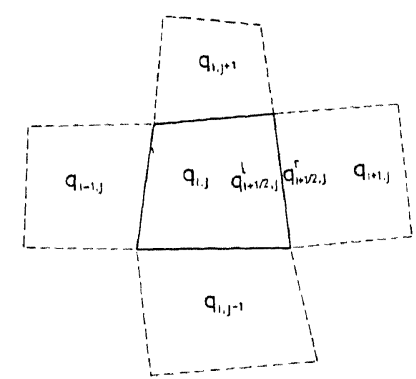

(b)

Figure 1. Finite volume $\Omega_{i, j}$ : (a) geometry; (b) state vectors

and similar expressions for $\mathbf{F}_{i-1 / 2, j}, \mathbf{F}_{i, j+1 / 2}$ and $\mathbf{F}_{i, j-1 / 2} \cdot \mathbf{F}_{i+1 / 2, j}$ represents the transport of mass, momentum and energy per unit of time across $\partial \Omega_{i+1 / 2, j}$. In $(10), l_{i+1 / 2, j}$ is the length of the finite volume wall $\partial \Omega_{i+1 / 2, j}$, and the superscripts $l$ and $r$ refer to the left and right side of $\partial \Omega_{i+1 / 2, j}$ respectively (Figure 1(b)).

By considering the flux vector to be determined by a uniformly constant left and right state only, the actual flux evaluation is identical to the solution of a ID Riemann problem. For this we use here a so-called approximate Riemann solver. Several approximate Riemann solvers exist. ${ }^{3-6} \mathrm{We}$ have chosen Osher's Riemann solver because of (i) its continuous differentiability and (ii) its consistent treatment of boundary conditions. ${ }^{7,8}$ (The continuous differentiability guarantees the applicability of a Newton-type solution technique, which is what we make use of.) Osher's scheme is generally said to be complicated and expensive compared to other approximate Riemann solvers. Arguments against this can be found in Reference 8 .

The approximate solution of the 1D Riemann problem is called the evolution stage of a socalled projection-evolution scheme. ${ }^{9}$ Still to be filled in is the projection stage, i.e. the determination of the left and right states, such as $\mathbf{q}_{i+1 / 2, j}^{l}$ and $\mathbf{q}_{i+1 / 2, j}^{r}$ in (10). Depending on the way the states $\mathbf{q}_{i+1 / 2, j}^{l}$ and $\mathbf{q}_{i+1 / 2, j}^{r}$ are chosen, the discretization is first- or second-order accurate. First-order accuracy is simply obtained by taking

$$
\mathbf{q}_{i+1 / 2, j}^{l}=\mathbf{q}_{i, j}, \quad \mathbf{q}_{i+1 / 2, j}^{r}=\mathbf{q}_{i+1, j} .
$$

Second-order accuracy can be obtained by, for example, the $k$-schemes introduced by van Leer. ${ }^{9}$ Two well known drawbacks of the first-order accurate discretization are (i) its need for relatively fine grids in smooth flow regions and (ii) its strong smearing of discontinuities that are not aligned with the grid. ${ }^{10}$

Second-order discretizations yield a strong improvement of both drawbacks. However, secondorder discretized equations cannot be solved with the same good efficiency as first-order discretized equations. ${ }^{11}$ Further, when using a second-order discretization, spurious nonmonotonicity (wiggles) may arise at discontinuities. ${ }^{11}$

Here we prefer the first-order accurate discretization, since (i) the transonic shock will be well aligned with the grid and (ii) the best possible efficiency is preferred.

\subsection{Solution method}

2.2.1. Relaxation method. As a solution method for the first-order discretized equations we use collective symmetric point Gauss-Seidel relaxation. Point refers to the fact that during the update 
of the state vector $\mathbf{q}_{i, j}$ all other state vectors are kept fixed. Collective refers to the fact that the update of $\mathbf{q}_{i, j}$ is done for all of its four components simultaneously. Further, symmetric means that after a relaxation sweep, i.e. after an update of all state vectors $\mathbf{q}_{i, j}$, a new sweep in the reverse direction is made. At each volume visited during a relaxation sweep we solve the four non-linear equations (9) by Newton's method (local linearization). The most efficient relaxation is obtained by selecting a large tolerance for the Newton iteration so that in all but exceptional cases only a single Newton step is needed.

2.2.2. Multigrid method. The solution method described so far is simple and robust but needs an acceleration. With point Gauss-Seidel relaxation a suitable acceleration technique is found in multigrid. As a very efficient and robust multigrid technique we use non-linear multigrid preceded by nested iteration. 8,12

Let

$$
F_{h}\left(q_{h}\right)=0
$$

denote the system of first-order discretized Euler equations. To apply multigrid, we construct a nested set of grids such that each volume on a coarse grid is the union of $2 \times 2$ volumes on the next finer grid (Figure 2).

Let $\Omega_{h_{1}}, \Omega_{h_{2}}, \ldots, \Omega_{h_{L}}$ be a sequence of nested grids with $\Omega_{h_{1}}$ the coarsest and $\Omega_{h_{L}}$ the finest grid. Our multigrid solution of (12) can be divided into two successive stages. The first stage is nested iteration (or full multigrid) which is used to obtain a good initial solution on $\Omega_{h_{L}}$. The second and last stage is non-linear multigrid (or full approximation scheme) which is used to iterate until convergence. The first iterand for the non-linear multigrid iteration is the solution obtained by nested iteration. We will now discuss these stages in more detail.

Nested iteration. The nested iteration starts with a user-defined initial estimate of $q_{h_{1}}$, the solution on the coarsest grid. To obtain an initial solution on a finer grid $\Omega_{h_{l+1}}$, first the solution on the next coarser grid $\Omega_{h_{l}}$ is improved by a non-linear multigrid cycle. Hereafter this solution is interpolated to the finer grid $\Omega_{h_{l+1}}$. These steps are repeated until the highest level (finest grid) has been reached. On a grid $\Omega_{h_{1}}$ with an even number of volumes in both the $i$ - and $j$-direction, the interpolation used to obtain the initial solution on a next finer grid is bilinear. For this purpose the grid $\Omega_{h_{l}}$ is subdivided into disjunct sets of $2 \times 2$ volumes. The four state vectors corresponding with each set are interpolated in a bilinear way. Since each volume of $\Omega_{h_{l}}$ overlaps $2 \times 2$ volumes of $\Omega_{h_{l+1}}, 4 \times 4$ new state vectors are obtained on $\Omega_{h_{l+1}}$. (On a coarsest grid with an odd number of volumes in the $i$ - and/or $j$-direction, the interpolation used is piecewise constant).

Non-linear multigrid iteration. A single non-linear multigrid cycle is recurrently defined by the following steps:

1. Improve on $\Omega_{h_{l}}$ the latest obtained solution $q_{h_{l}}$ by application of $p$ pre-relaxation sweeps.

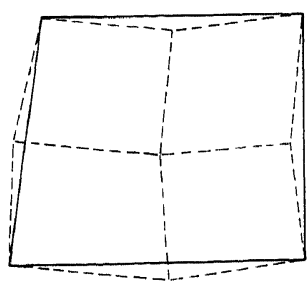

Figure 2. Coarse grid volume and corresponding fine grid volumes 
2. Compute on the next coarser grid $\Omega_{h_{l-1}}$ the right-hand side $r_{h_{l-1}}=F_{h_{l-1}}\left(q_{h_{l-1}}\right)-I_{h_{l}}^{h_{l-1}} F_{h_{l}}\left(q_{h_{l}}\right)$, where $I_{h_{1}}^{h_{l}-1}$ is a so-called restriction operator.

3. Approximate the solution of $F_{h_{l-1}}\left(q_{h_{l-1}}\right)=r_{h_{l-1}}$ by application of $\sigma$ non-linear multigrid cycles. Denote the approximation obtained as $\tilde{q}_{h_{1-1}}$.

4. Correct the current solution by $q_{h_{l}}=q_{h_{l}}+I_{h_{l-1}}^{h_{l}}\left(\tilde{q}_{h_{l-1}}-q_{h_{l-1}}\right)$, where $I_{h_{l-1}}^{h_{l}}$ is a so-called prolongation operator.

5. Improve again $q_{h_{l}}$ by application of $q$ post-relaxations.

Steps 2, 3 and 4 form the so-called coarse grid correction. (These three steps are skipped on the coarsest grid.) Notice that besides the discrete operator, the boundary condition treatment is also the same on all grid levels. The efficiency of a coarse grid correction depends in general on the coarseness of the coarsest grid (in general: the coarser, the better).

The restriction operator $I_{h_{l}-1}^{h_{l}}$ and the prolongation operator $I_{h_{l-1}}^{h_{l}}$ are defined by

$$
\begin{gathered}
\left(r_{h_{l-1}}\right)_{i, j}=\left(I_{h_{l}}^{h_{l-1}} r_{h_{l}}\right)_{i, j} \equiv\left(r_{h_{l}}\right)_{2 i, 2 j}+\left(r_{h_{l}}\right)_{2 i-1,2 j}+\left(r_{h_{l}}\right)_{2 i, 2 j-1}+\left(r_{h_{l}}\right)_{2 i-1,2 j-1}, \\
\left(I_{h_{l-1}}^{h_{l}} q_{h_{l-1}}\right)_{2 i, 2 j}=\left(I_{h_{l-1}}^{h_{l}} q_{h_{l-1}}\right)_{2 i-1,2 j}=\left(I_{h_{l-1}}^{h_{l}} q_{h_{l-1},}\right)_{2 i, 2 j-1}=\left(I_{h_{l-1}}^{h_{l}} q_{h_{l-1}}\right)_{2 i-1,2 j-1} \equiv\left(q_{h_{l-1}}\right)_{i, j} .
\end{gathered}
$$

Defining the transfer operators in this way, it can be verified that

$$
F_{h_{l-1}}=I_{h_{l}}^{h_{l-1}} F_{h_{l}} I_{h_{l-1}}^{h_{l}} \text {, }
$$

i.e. a coarse grid discretization of the Euler equations is a Galerkin approximation of the discretization on the next finer grid. This implies that the coarse grid correction reduces in an efficient way the low-frequency components in the defect.

As values for $\sigma, p$ and $q$ we generally use at each level separately $\sigma=1$ and $p=q=1$; i.e. as nonlinear multigrid cycles we generally use $\mathrm{V}$-cycles with one pre- and one post-relaxation.

The complete solution process does not need any tuning. In Figure 3 an illustration is given of the structure of the multigrid method. A five-level multigrid strategy has been considered. Between each pair $\mathrm{AB}$ we have a non-linear multigrid cycle (V-cycle). In the nested iteration stage, between each pair BA we have the bilinear prolongation of the solution. For an account of the computational efficiency of the present and other methods we refer to Reference 13.

\section{GRID}

In Figure 4 graphs are given of the wind tunnel section considered. In Figure 4(a) a graph is given of the complete integration region. The graph shows a flat parallel inflow part, followed by a slender curved part up to the outlet. In Figure 4(b) a photograph is given of the test section in an opened wind tunnel.

In order to obtain a good resolution of large local gradients (which we decided were important), we used grids with local refinements; grids with stretching in both the $x$-and $y$-direction (Figure 5).

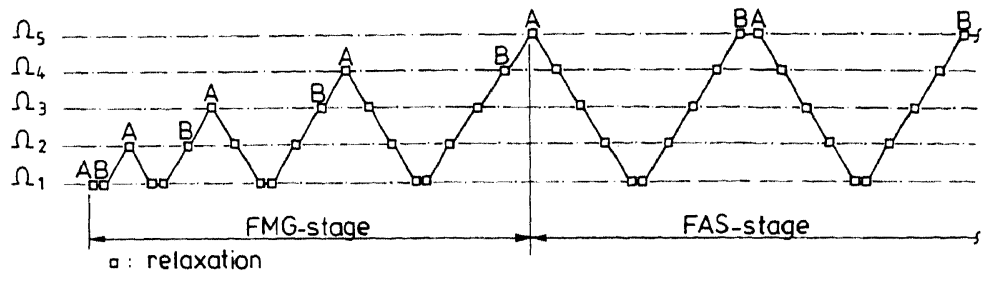

Figure 3. Schematic representation of a (five-level) multigrid strategy 


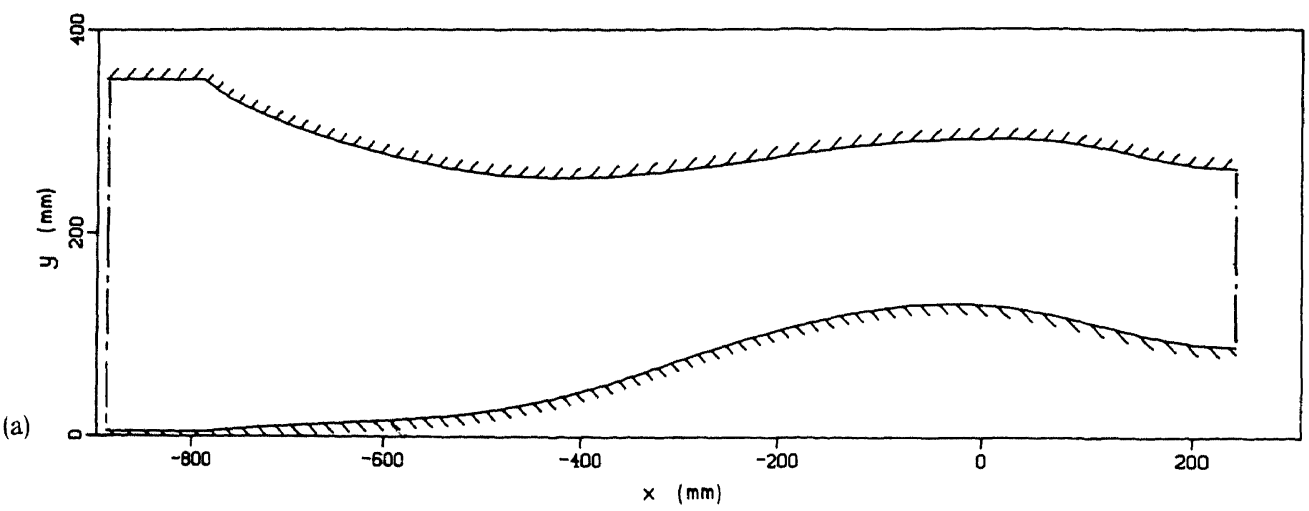

b)

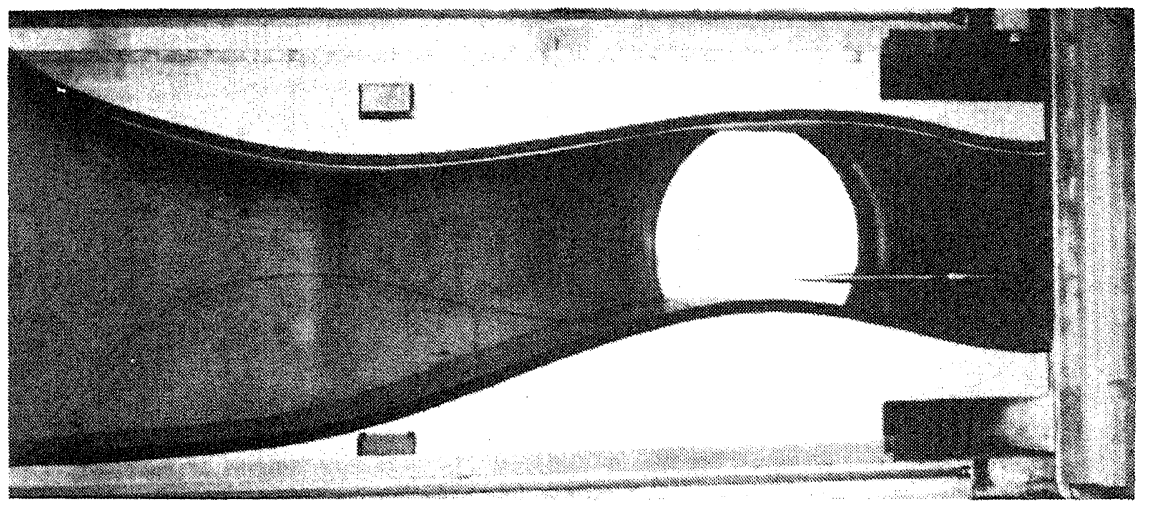

Figure 4. Wind tunnel section: (a) complete integration region; (b) test section in opened wind tunnel

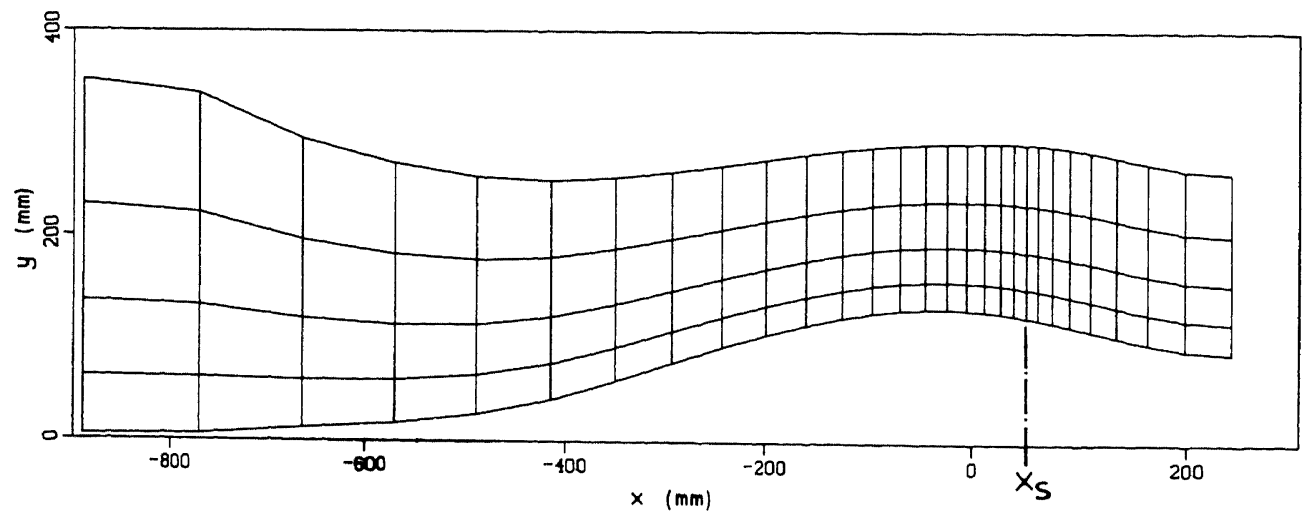

Figure 5. Stretched grid

In the $x$-direction the grid is most refined at $x_{\mathrm{s}}$. The value of $x_{\mathrm{s}}$ is adapted during the solution process. It is initialized to the $x$-location of the wind tunnel throat and adapted to the shock location during the nested iteration stage. The grid adaptation is simple. First, after each solution prolongation in the nested iteration stage, a search is made for the $x$-location of the maximum 
velocity gradient at the lower wall, downstream of the throat. This location is assigned to $x_{\mathrm{s}}$. Hereafter, we generate the new grids. Without any correction, the states $\mathbf{q}_{i, j}$ are shifted together with the volumes $\Omega_{i, j}$. In doing this, the quality of the finest grid solution as yielded by the nested iteration becomes worse. However, no significant deterioration of convergence rates has been observed. (The grid adaptation has no other effect than that for which it is intended, i.e. to reduce the mesh size at the shock wave in order to make it sharper.)

\section{BOUNDARY CONDITIONS}

\subsection{Different houndary conditions considered}

The boundary condition treatment must be correct both mathematically and physically. Mathematics prescribes how many conditions must be imposed at a boundary; physics prescribes what conditions should be imposed.

The number of conditions to be imposed at a boundary depends on the type of flow at that boundary. Types of flow to be considered here (and the corresponding number of boundary conditions to be imposed) are (i) subsonic inflow (three), (ii) subsonic outflow (one) and (iii) impermeable walls (one). The following conditions have been imposed.

Upper and lower wall. At the impermeable upper and lower wall the boundary condition imposed is trivial: a zero normal velocity component.

Inlet. Uniformly constant distributions $u=u_{\mathrm{in}}, v=0$ and $c=1$, with $c$ denoting the speed of sound, have been imposed. These distributions are motivated by the fact that the inlet part is flat and parallel. The $1 \mathrm{D}$ flow theory value, given a sonic throat, has been taken as a subsonic value for $u_{\text {in }}$.

Outlet. Because of the fact that the outlet part is non-flat and non-parallel, the outlet boundary condition cannot be as trivial as those at the inlet. The following possibilities have been considered: (i) $h=h_{\text {in }}$ is uniformly constant, with $h$ denoting the total enthalpy $h=c^{2} /(\gamma-1)+\frac{1}{2}\left(u^{2}+v^{2}\right)$; (ii) $v / u=\theta(y)$; (iii) $u=u(y)$; (iv) $p=p(y)$.

The first possibility was motivated by the fact that with a known uniformly constant distribution of $u, v$ and $c$ at the inlet (i.e. with a known uniformly constant total enthalpy at the inlet), this boundary condition requires no knowledge of the non-uniform outlet flow. This because of the fact that for steady 2D Euler flows, with the total enthalpy at the upstream boundary known to be constant, only three differential equations describe the flow. The energy equation in its differential form may be replaced by the relation $c^{2} /(\gamma-1)+\frac{1}{2}\left(u^{2}+v^{2}\right)=h_{\text {in }}$ throughout the entire flow field. The present Euler code solves the full non-isenthalpic Euler equations. To allow the computation of non-isenthalpic Euler flows such as the flow through a propeller disc, ${ }^{14}$ and in particular to allow a rapid extension to a Navier-Stokes code, ${ }^{15,16}$ the simplifying property mentioned has not been exploited.

The second possibility, with the flow direction specified, was motivated by its simplicity. A linear distribution of $\theta(y)$ has been assumed, using the known flow direction at the lower and upper wall.

The third possibility was also motivated by its simplicity. For this possibility we assumed the outlet flow to be a potential vortex flow. The relation $u(y) r(y)=\psi_{\text {out }}$ has been applied, with $\psi_{\text {out }}$ uniformly constant and $r(y)$ the distribution of the radii of curvature of the streamlines. A linear distribution for the streamline curvature $1 / r(y)$ has been assumed, using the known curvature of the lower and upper wall. The value following from 1D flow theory, given a sonic throat and a transonic outflow with a shock of known constant strength, has been taken as a value for $\psi_{\text {out }}$. A disad vantage of this boundary condition is its inconsistency in the case of a flow with a shock wave 
of variable strength, which is what we have here. (It is a boundary condition which is always consistent in a potential flow model, but not in the Eulerian rotational flow model.)

For the fourth possibility we used the equation of curvilinear motion

$$
\frac{\mathrm{d} p(y)}{\mathrm{d} y}=\gamma \frac{p(y) M^{2}(y)}{r(y)} \cos \phi(y),
$$

with $M(y)$ the Mach number distribution and $\phi(y)$ the distribution of the angles between the streamlines and the $x$-axis. For $1 / r(y)$ and $\phi(y)$ linear distributions have again been assumed, such that the flow fits the channel outlet. $M(y)$ has been taken uniformly constant. Its value has been determined from 1D flow theory, again given a sonic throat and a transonic outflow with a shock of known constant strength. Using the corresponding value of $p$ as the value for $p$ at the lower wall, an initial value problem was obtained, which has been solved by means of a RungeKutta-Merson method. In the next section the well-posedness of each of these four outlet boundary conditions is investigated. There a choice is made for the outlet boundary condition to be applied.

\subsection{Well-posedness subsonic outlet boundary conditions}

Generally speaking, mathematically well-posed conditions to be imposed at a boundary are conditions for which the state at that boundary can be completed accurately. At a subsonic outlet the boundary condition must fix the single degree of freedom existing overthere. A subsonic outlet boundary condition can be represented as a 3D surface in a 4D state space. The smaller the angle $\alpha$ between the normal at this surface and the eigenvector corresponding to the negative eigenvalue of the Jacobian, $u-c$, the better the quality of the outlet boundary condition. Considering the $(\rho, u, v, e)$-space as state space, the eigenvector corresponding to the eigenvalue $u-c$ is $\mathbf{r}=(\rho,-c, 0, c(c / \gamma-u))^{\mathrm{T}}$. For $h, v / u, u$ and $p$ specified respectively, the 3D surface mentioned, say $B(q)$, is described by

$$
\begin{aligned}
& B(q)=\gamma e-\frac{\gamma-1}{2}\left(u^{2}+v^{2}\right)=h_{B}, \\
& B(q)=v / u=\theta_{B}, \\
& B(q)=u=u_{B}, \\
& B(q)=(\gamma-1) \rho\left[e-\frac{1}{2}\left(u^{2}+v^{2}\right)\right]=p_{B},
\end{aligned}
$$

with $h_{B}, \theta_{B}, u_{B}$ and $p_{B}$ constant. For the angle $\alpha$ it holds that $\cos \alpha \propto \nabla B \cdot \mathbf{r}$, with $\nabla=(\partial / \partial \rho, \partial / \partial u$, $\partial / \partial v, \partial / \partial e)^{\mathrm{T}}$. For $h, v / u, u$ and $p$ specified respectively, we find

$$
\begin{aligned}
& \nabla B \cdot \mathbf{r}=c(c-u), \\
& \nabla B \cdot \mathbf{r}=-(v / u)(c / u), \\
& \nabla B \cdot \mathbf{r}=-c, \\
& \nabla B \cdot \mathbf{r}=\gamma p .
\end{aligned}
$$

From (18a) and (18b) it can be seen that for $h$ and $v / u$ specified, the vectors $\nabla B$ and $\mathbf{r}$ become orthogonal for $u \rightarrow c$ and $v \rightarrow 0$ respectively. The consequence of a nearly orthogonal $\nabla B$ and $\mathbf{r}$ is that a small change in either the boundary condition or the state inside the integration region near the outlet may cause a large change in the boundary state and hence in the flux across the outlet. 
For a given state $\mathbf{q}_{0}=\left(u_{0}, v_{0}, c_{0}, z_{0}\right)^{\mathrm{T}}$ near the outlet, with $z_{0}=\ln \left(p_{0} \rho_{0}^{-\gamma}\right)$, and the boundary conditions specified by (17a)-(17d) respectively, the effect of a perturbation in $\mathbf{q}_{0}$ will be shown.

The state at the outlet is $\mathbf{q}=\left(u, v_{0}, c, z_{0}\right)^{\mathrm{T}}$, with, for boundary conditions (17a)-(17d) respectively,

$$
\begin{aligned}
& c=\frac{\gamma-1}{\gamma+1}\left\{u_{0}+\frac{2}{\gamma-1} c_{0}+\sqrt{ }\left[(\gamma+1)\left(h_{B}-\frac{1}{2} v_{0}^{2}\right)-\frac{\gamma-1}{2}\left(u_{0}+\frac{2}{\gamma-1} c_{0}\right)^{2}\right]\right\}, \\
& u=v_{0} / \theta_{B}, \\
& u=u_{B}, \\
& c=\sqrt{ }\left(\gamma p_{B}^{(\gamma-1) / \gamma} e^{z_{0} / \gamma}\right)
\end{aligned}
$$

and either

$$
u=u_{0}+\frac{2}{\gamma-1}\left(c_{0}-c\right) \quad \text { or } \quad c=c_{0}+\frac{\gamma-1}{2}\left(u_{0}-u\right)
$$

to complete.

With $\nabla=\left(\partial / \partial u_{0}, \partial / \partial v_{0}, \partial / \partial c_{0}, \partial / \partial z_{0}\right)^{\mathrm{T}}$ we find for $(19 \mathrm{a})-(19 \mathrm{~d})$ respectively

$$
\begin{aligned}
& \nabla c=\frac{\gamma-1}{\gamma+1}\left(1,0, \frac{2}{\gamma+1}, 0\right)^{\mathrm{T}}-\frac{\gamma-1}{\gamma+1} \frac{1}{c-u}\left(\frac{\gamma-1}{2} u+c, \frac{\gamma+1}{2} v, u+\frac{2}{\gamma-1} c, 0\right)^{\mathrm{T}}, \\
& \nabla u=(0, u / v, 0,0)^{\mathrm{T}}, \\
& \nabla u=\mathbf{0}, \\
& \nabla c=(0,0,0, c / 2 \gamma)^{\mathrm{T}},
\end{aligned}
$$

where we have substituted $\mathbf{q}=\mathbf{q}_{0}$ into (20a) and (20b) for simplicity. It can be seen that the gradient pairs (20a) and (20b) become infinitely large in the aforementioned limit cases $(u \rightarrow c$ for $h$ specified and $v \rightarrow 0$ for $v / u$ specified). Flow computations with $h$ specified and $v / u$ specified showed these outlet boundary conditions to be ill-posed indeed. This was not the case with the two other boundary conditions.

Because of its better consistency with the Euler flow model, the boundary condition with $p$ specified has been preferred to the boundary condition with $u$ specified.

\section{RESULTS}

The great slenderness of the wind tunnel section led to a coarsest grid with a relatively large number of volumes in the longitudinal direction. By using a $56 \times 8$ grid as the finest grid and a four-level multigrid strategy, a $7 \times 1$ grid was the coarsest grid (Figure 6). (The dashed lines in Figure 6 indicate the lower and upper wall of the wind tunnel section.)

As a consequence of this relatively fine coarsest grid, the number of non-linear multigrid cycles required was somewhat larger than the few cycles which are supposed to be necessary for optimal multigrid efficiency. To slightly improve the multigrid convergence properties, we increased the number of pre- and post-relaxations performed on the coarsest grid.

As flow problems we considered a non-choked flow with $M_{\mathrm{u}}=1 \cdot 15, M_{\mathrm{u}}$ being the Mach number just upstream of the shock wave, and a choked flow with $M_{\mathrm{u}}=1 \cdot 37$.

Convergence histories obtained for both test cases are given in Figure 7. In both convergence histories the single-grid history is given as well. The convergence histories are given by graphs of the residual ratio $\Sigma_{i=1}^{4}\left|r_{h}^{n}(i)\right|_{\max } / \Sigma_{i=1}^{4}\left|r_{h}^{1}(i)\right|_{\max }$ versus the number of work units. Here $\left|r_{h}^{n}(i)\right|_{\max }$ 
(a)

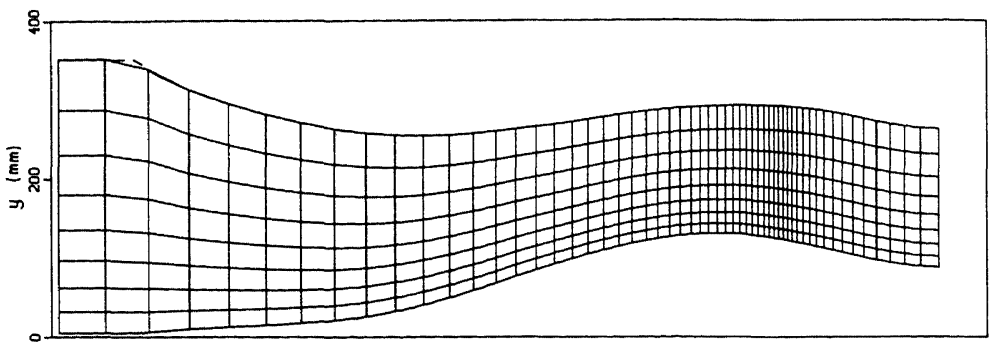

(b)

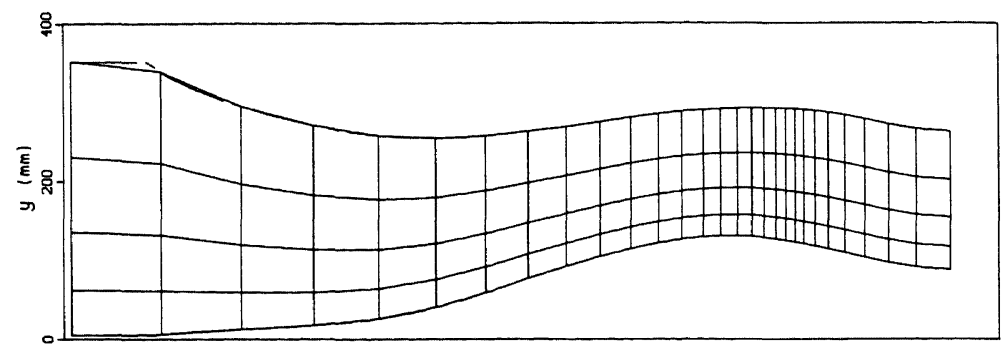

(c)

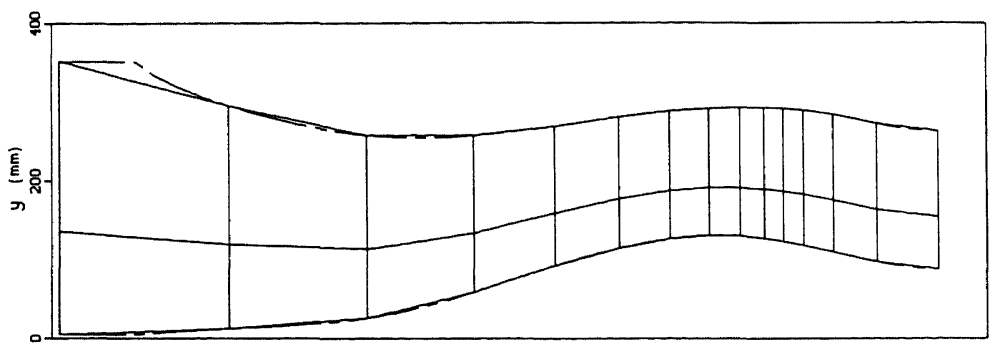

(d)

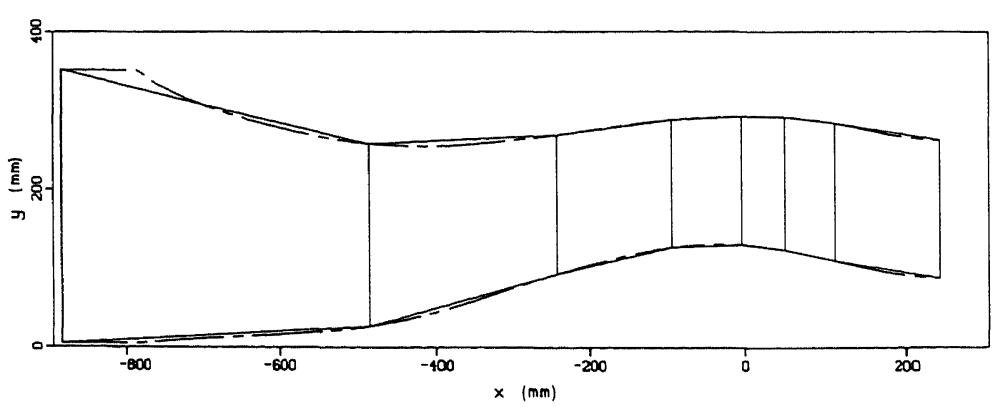

Figure 6. Family of grids: (a) $56 \times 8$ grid; (b) $28 \times 4$ grid; (c) $14 \times 2$ grid; (d) $7 \times 1$ grid

denotes the maximum absolute value over all volumes of the $i$ th component of the residual $r_{h}^{n}=F_{h}\left(q_{h}^{n}\right)$, and $n$ denotes the $n$th work unit. A work unit is defined as one multigrid cycle, and for the single-grid computation as the equivalent number of fine grid relaxation sweeps. (The rather poor multigrid acceleration obtained for the choked flow may have been caused by too low an accuracy of the prolongation operator $I_{h_{l-1}}^{h_{l}}$.)

Finest grids and lower surface pressure distributions obtained for $M_{\mathrm{u}}=1.15$ and $M_{\mathrm{u}}=1.37$ are shown in Figure 8. The open circles in the pressure distributions correspond to computed pressures, the full circles to measured pressures. A very satisfactory agreement is of course found 

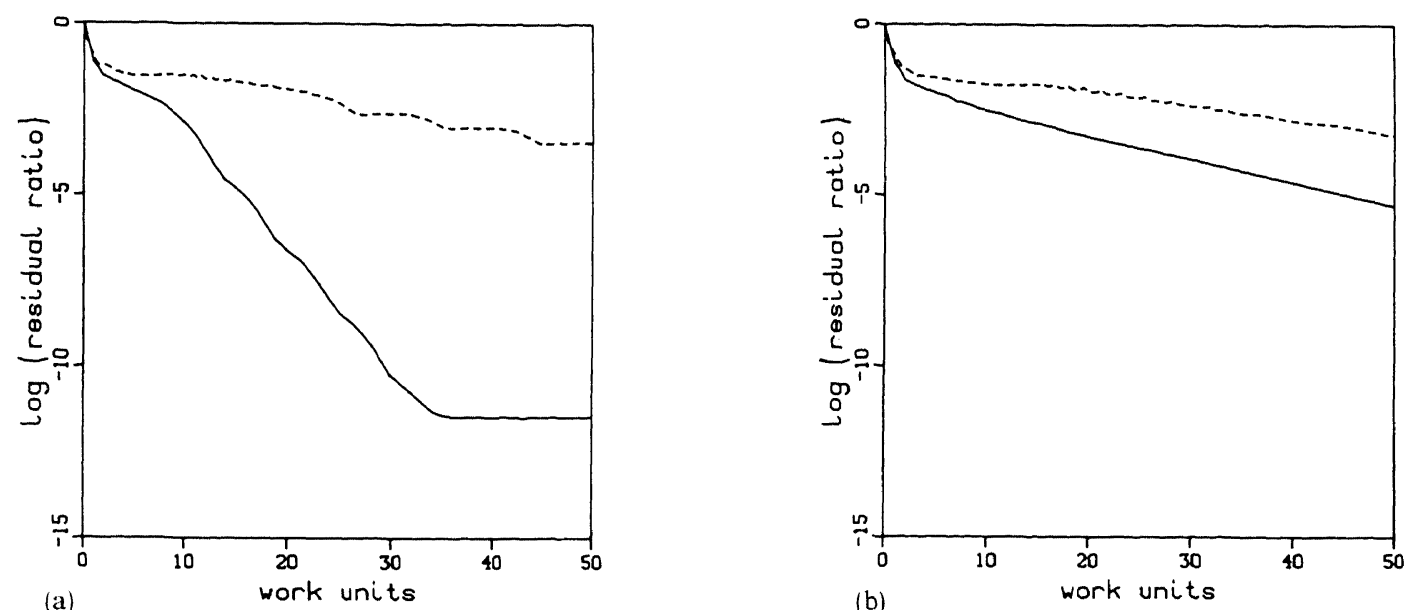

Figure 7. Convergence histories (- multigrid; ------, single grid): (a) $M_{\mathrm{u}}=1 \cdot 15 ;$ (b) $M_{\mathrm{u}}=1 \cdot 37$

away from the wall and shock wave. Yet an important result - the pressure rise across the shock wave at the wall-is also predicted in a satisfactory way. The latter indicates that the Euler code may be exploited for designing experimental set-ups like this. From the finest grids and surface distributions obtained, it can be seen that for both flows the grid adaptation is good. Clearly visible for $M_{\mathrm{u}}=1.37$ is the occurrence of an after-expansion. Since a first-order accurate Oshertype discretization yields solutions without spurious non-monotonicity, the after-expansion occurring for $M_{\mathrm{u}}=1.37$ is not a numerical artefact but indeed a correct part of the Euler flow solution.

Further, for both the choked and non-choked flow, a comparison is made between the Mach number distributions obtained with the Euler code and those obtained by holographic interferometry. In Figure 9 the Mach number distributions are given as obtained for the entire test section. In Figure 10 a detail of both distributions is compared with the corresponding interferometric result. It appears that the computational and experimental results show a perfect quantitative agreement away (of course) from the wall and shock wave.

The differences between computational and experimental results can also be exploited. Given an Euler code which has proved to be reliable, its results can be considered confidently as experimental results with viscosity and heat conduction switched off. Its results can be used for identifying simple viscous phenomena and, in particular, complicated viscous-inviscid phenomena. The present Euler code has proved to be reliable. ${ }^{11,13,14}$ Here it shows for instance that the downstream supersonic pocket and the $\lambda$-shock (at $M_{\mathrm{u}}=1 \cdot 37$ ) are indeed viscous-inviscid phenomena and that the shock wave has been displaced slightly upstream by viscous effects.

\section{CONCLUSIONS}

For the steady 2D non-isenthalpic Euler equations, an outlet boundary condition with total enthalpy or flow direction specified yields a mathematically ill-posed problem, whereas an outlet boundary condition with static pressure specified yields a mathematically well-posed problem.

The convergence rates obtained are not optimal from the viewpoint of multigrid techniques, though still very good from the viewpoint of almost any other solution technique. The multigrid convergence behaviour suffered somewhat from the relatively large number of volumes in the streamwise direction on the coarsest grid. 

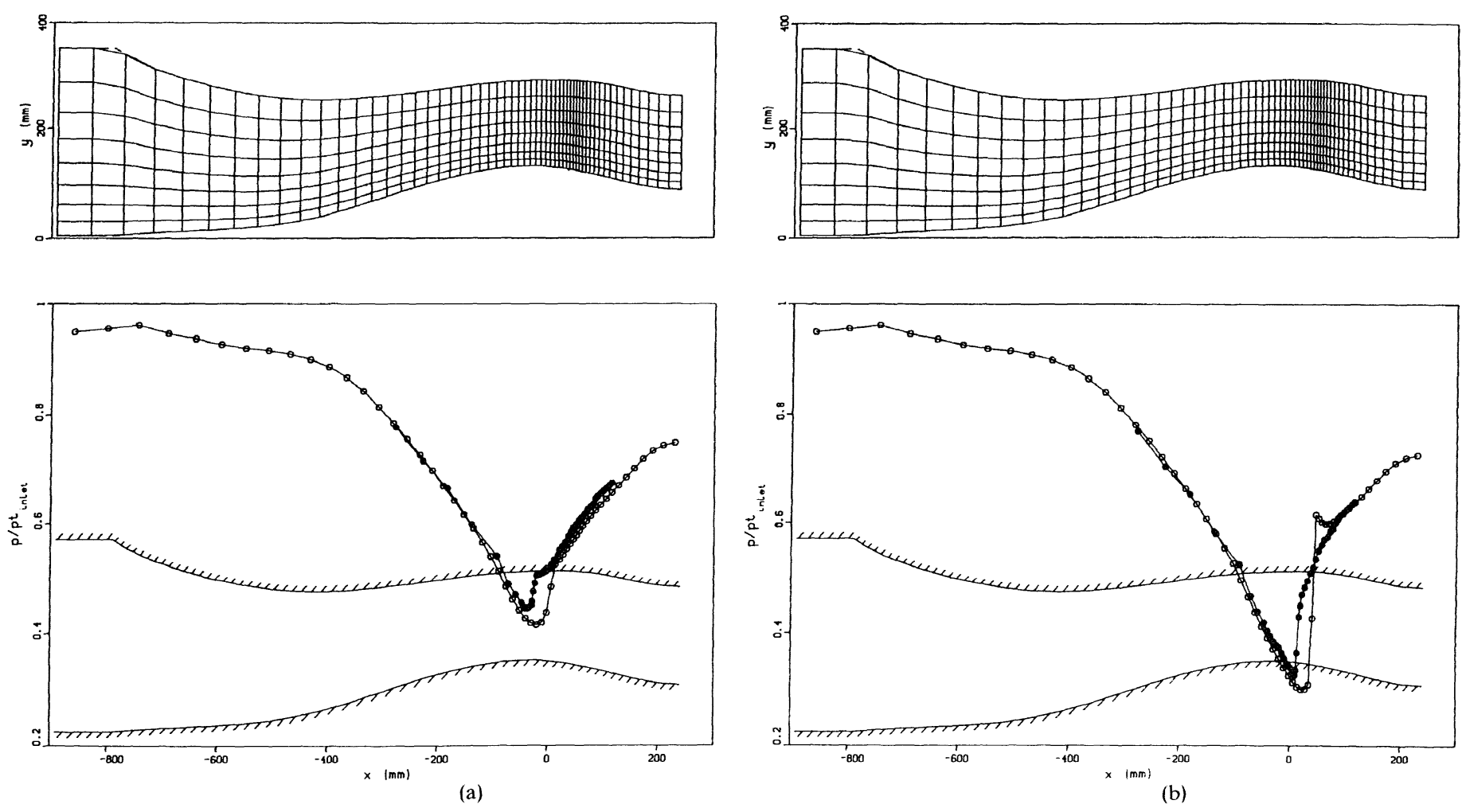

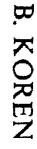

Figure 8. Grids and lower surface pressure distributions ( $\bigcirc$, computed; $\bullet$, measured): (a) $M_{\mathrm{u}}=1 \cdot 15$; (b) $M_{\mathrm{u}}=1 \cdot 37$ 


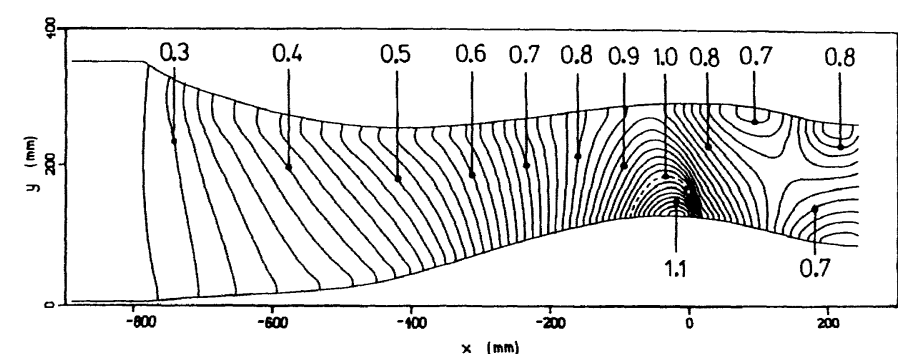

(a)

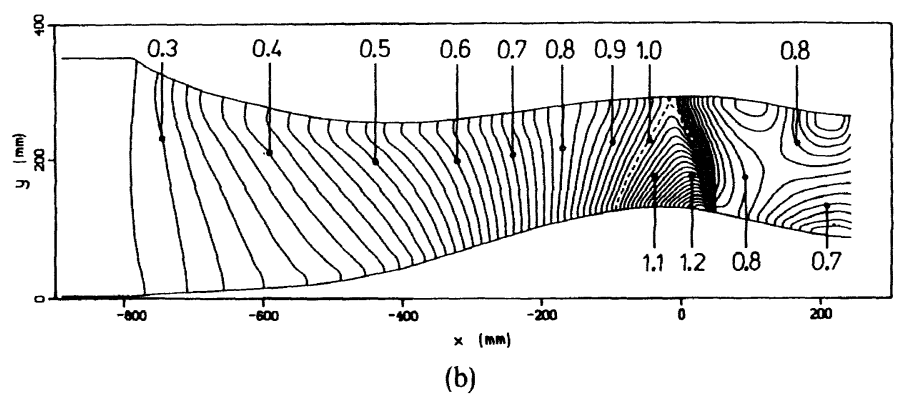

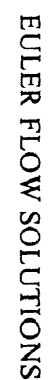

Figure 9. Mach number distributions: (a) $M_{\mathrm{u}}=1 \cdot 15$; (b) $M_{\mathrm{u}}=1 \cdot 37$ 

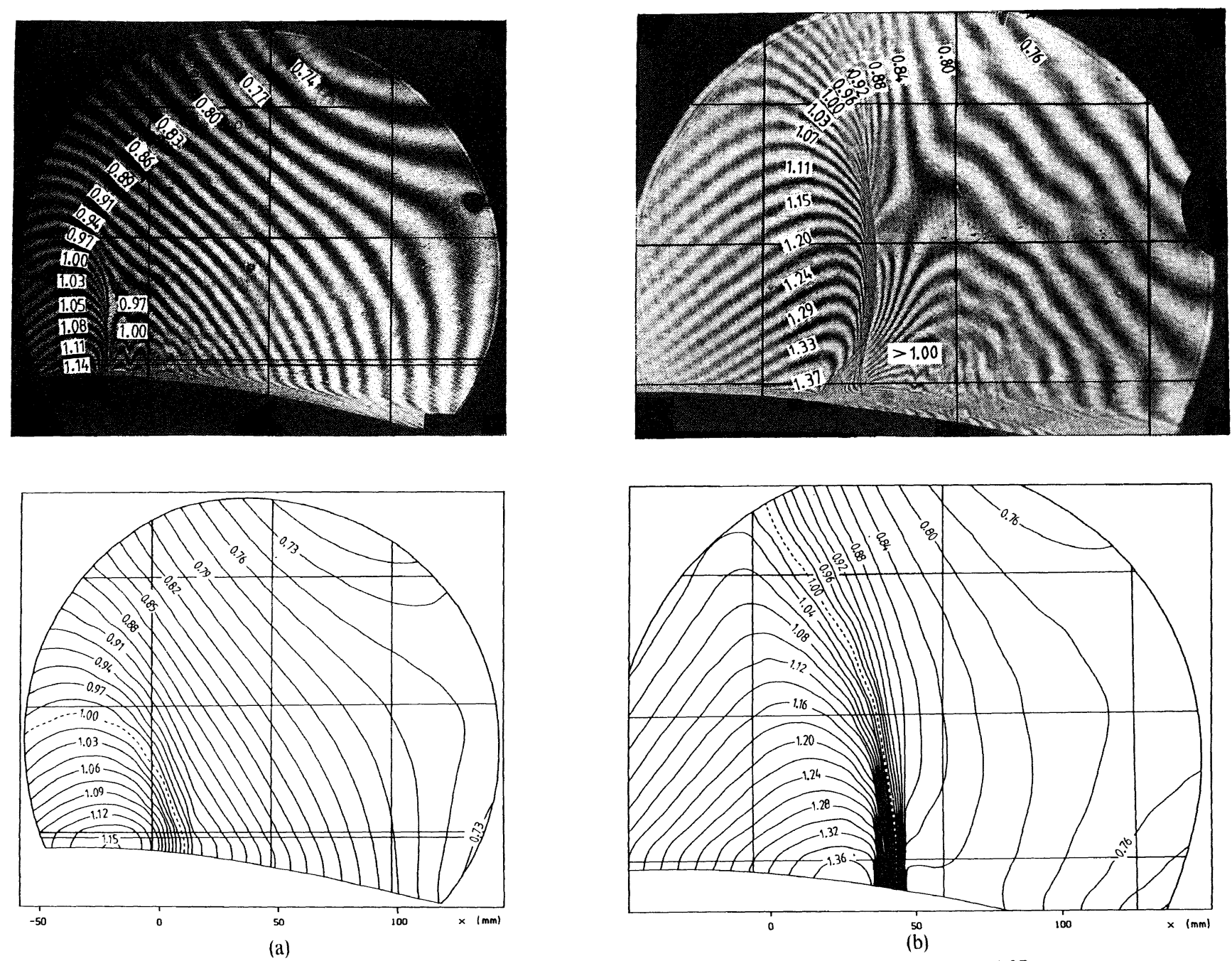

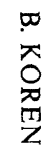

Figure 10. Interferometric and numerical Mach number distributions: (a) $M_{\mathrm{u}}=1 \cdot 15$; (b) $M_{\mathrm{u}}=1 \cdot 37$ 
Non-oscillatory solutions with sharp shocks have been obtained. Automatic grid adaptation during the nested iteration works well. Away from the walls and shock wave, the agreement between computational and experimental results is good. A main advantage of the solution method is that it is parameter-free; it needs no tuning.

The usefulness of a reliable Euler code in research on viscous-inviscid interactions is twofold. It may be used (i) as a tool for designing (and redesigning) an experimental set-up and (ii) as a tool for understanding complicated experimental results. Given the recent availability of very reliable (and, moreover, very efficient) Euler codes, this use might become important in near-future research.

\section{ACKNOWLEDGEMENT}

This work was supported by the Netherlands Technology Foundation (STW).

\section{REFERENCES}

1. C. Nebbeling and W. J. Bannink, 'A curved test section for research on transonic shock wave-boundary layer interaction', Report LR-414, Delft University of Technology, 1984.

2. C. Nebbeling and B. Koren, 'An experimental-computational investigation of transonic shock wave-turbulent boundary layer interaction in a curved test section', Report NM-R8716, Centre for Mathematics and Computer Science, Amsterdam, 1987.

3. J. L. Steger and R. F. Warming. 'Flux-vector splitting of the inviscid gas dynamic equations with applications to finitedifference methods', J. Comput. Phys., 40, 263-293 (1981).

4. B. van Leer, 'Flux-vector splitting for the Euler equations', Proc. 8th Int. Conf. on Numerical Methods in Fluid Dynamics, Aachen, 1982; Lecture Notes in Physics, Vol. 170, Springer, Berlin, 1982, pp. 507-512.

5. P. L. Roe, 'A pproximate Riemann solvers, parameter vectors, and difference schemes', J. Comput. Phys., 43, 357-372 (1981).

6. S. Osher and F. Solomon, 'Upwind difference schemes for hyperbolic systems of conservation laws', Math. Comput., 38, 339-374 (1982)

7. S. Osher and S. Chakravarthy, 'Upwind schemes and boundary conditions with applications to Euler equations in general geometries', $J$ '. Comput. Phys., 50, 447-481 (1983).

8. P. W. Hemker and S. P. Spekreijse, "Multiple grid and Osher's scheme for the efficient solution of the steady Euler equations', Appl. Numer. Math., 2, 475-493 (1986).

9. B. van Leer, 'Upwind-difference methods for aerodynamic problems governed by the Euler equations', Proc. 15th AMS-SI AM Summer Seminar on Applied Mathematic's, Scripps Institution of Oceanography, 1983; Lectures in Applied Mathematics, Vol. 22, AMS, Providence, Rhode Island, 1985, pp. 327-336.

10. P. W. Hemker, 'Defect correction and higher order schemes for the multi grid solution of the steady Euler equations', Proc. 2nd European Conf. on Multigrid Methods, Cologne, 1985; Lecture Notes in Mathematics, Vol. 1228, Springer, Berlin, 1986, pp. 150-165.

11. B. Koren, 'Defect correction and multigrid for an efficient and accurate computation of airfoil flows', J. Comput. Phys., 77, 183-206 (1988)

12. W. Hackbusch, Multi-Grid Methods and Applications, Springer, Berlin, 1985.

13. P. W. Hemker and B. Koren, 'A non-linear multigrid method for the steady Euler equations', to appear in Proc. GAMM Workshop on the Numerical Solution of Compressible Euler Flows, Rocquencourt, 1986; Notes on Numerical Fluid Mechanics, Vieweg, Braunschweig 1988.

14. B. Koren and S. P. Spekreijse, 'Multigrid and defect correction for the efficient solution of the steady Euler equations', Proc. 25th Meeting of the Dutch Association for Numerical Fluid Mechanics, Delft, 1986; Notes on Numerical Fluid Mechanics, Vol. 17, Vieweg, Braunschweig, 1987, pp. 87-100.

15. B. Koren, 'Multigrid and defect correction for the steady Navier-Stokes equations', to appear in Proc. Fourth GAMM-Seminar Kiel on Robust Multi-Grid Methods, Kiel, 1988; Notes on Numerical Fluid Mechanics, Vieweg, Braunschweig, 1988.

16. B. Koren, 'Upwind schemes for the Navier-Stokes equations', to appear in Proc. Second Int. Conf. on Hyperbolic Problems, Aachen, 1988; Notes on Numerical Fluid Mechanics, Vieweg, Braunschweig, 1988. 